\title{
Angiopoietin 1 and angiopoietin 2 are associated with medial thickening of hepatic arterial branches in biliary atresia
}

\author{
Aline F. de Souza ${ }^{1,2}$, Luise Meurer ${ }^{3,4}$, Themis R. da Silveira ${ }^{1,2}$, Cleandra Gregório ${ }^{2}$, Najara Reus², Carolina Uribe ${ }^{5}$, Ursula Matte ${ }^{1,5}$ \\ and Jorge L. dos Santos ${ }^{1,6}$
}

BACKGROUND: Biliary atresia (BA) is an infantile disorder characterized by progressive sclerosing cholangiopathy leading to biliary obstruction. First-line treatment of BA is hepatoportoenterostomy, the prognosis of which is related to age at surgery and to histological variables such as extent of fibrosis and ductular reaction. Hepatic arterial medial thickening (MT) suggests an arteriopathy in BA pathogenesis. We evaluated the expression of angiopoietin (ANGPT)/tyrosine kinase with immunoglobulin-like and epidermal growth factorlike domains 2 (TIE2) system in liver samples obtained from patients with BA, correlating it with MT, variables associated with disease severity, and postoperative prognosis.

METHODS: ANGPT1, ANGPT2, and TIE2 expression levels were assessed by quantitative PCR in liver samples obtained from BA patients $(n=23)$ at portoenterostomy and age-matched infants with intrahepatic cholestasis (IHC; $n=7)$. Histological variables were morphometrically assessed.

RESULTS: ANGPT1 and ANGPT2 were overexpressed in BA in comparison with $I H C(P=0.024$ and $P=0.029$, respectively). In BA, ANGPTs expression was positively correlated with MT (ANGPT1: $r_{\mathrm{s}}=0.59, P=0.013$; ANGPT2: $r_{\mathrm{s}}=0.52, P=0.032$ ), not with the variables associated with disease severity. TIE2 and ANGPTs expression levels were negatively correlated (ANGPT1: $r_{s}=-0.73, P<0.001$; ANGPT2: $r_{\mathrm{s}}=-0.54, P=0.007$ ).

CONCLUSION: In BA, there is overexpression of both ANGPT1 and ANGPT2, which is correlated with MT but not with age at portoenterostomy or with the histological variables associated with disease severity at the time of procedure.

B iliary atresia (BA) is an infantile hepatobiliary disorder characterized by the obstruction of bile ducts associated with a progressive sclerosing cholangiopathy. The primary treatment for BA is a hepatoportoenterostomy, which should be performed as soon as possible, because its chances of longterm success decrease when the age at surgery increases (1). In addition to age at portoenterostomy, histological findings observed in biopsies collected at the time of the procedure, such as the extents of fibrosis and ductular reaction, represent variables associated with disease severity and can help foresee the postoperative outcomes (2).

Regardless of portoenterostomy, BA induces cirrhosis by the continuous sclerosing cholangiopathy process, thus making this entity the leading cause of liver transplantation in children. The etiology of such a cholangiopathy remains elusive, and BA seems to represent more a phenotype than a unique disease. Our group is interested in the putative role of an arteriopathy in the development of BA because there are arteriographic (3), ultrasonographic (4-6), computed tomographic (7), histological (8), and immunohistochemical (9) evidences of medial thickening (MT) in hepatic artery branches owing to a vascular remodeling associated with hypoxia-ischemia in the portal tracts and porta hepatis. Knowledge on the behavior of angiogenic molecules in the liver of the affected patients can constitute an important element for understanding BA and its response to portoenterostomy. Therefore, the aim of this study was to evaluate the pattern of the angiopoietin (ANGPT)/tyrosine kinase with immunoglobulinlike and epidermal growth factor-like domains 2 (TIE2) system, molecules involved in vascular wall maturation (10), in the liver samples of patients with BA. These gene expression parameters were also correlated with the occurrence of MT, the behavior of clinical and histological variables associated with disease severity, and the postoperative outcomes. Our hypothesis was that ANGPT1 and its receptor TIE2 were overexpressed in the liver samples obtained from patients with BA in comparison with those in intrahepatic cholestasis (IHC) and that their expression levels in BA were positively correlated with MT and with variables associated with disease severity, affecting the postoperative outcomes.

\section{RESULTS}

This study included patients who underwent an exploratory laparotomy as part of the investigation of neonatal cholestasis from December 2006 to March 2011. The BA group comprised 7 boys and 16 girls aged 68 (range: 19-110) d of life at portoenterostomy, while the IHC group included 7 boys aged 81 (range: $39-147$ ) d of

'Department of Pediatrics, Universidade Federal do Rio Grande do Sul, Rio Grande do Sul, Brazil; ${ }^{2}$ Experimental Laboratory of Hepatology and Gastroenterology, Research Center, Hospital de Clínicas de Porto Alegre, Rio Grande do Sul, Brazil; ${ }^{3}$ Laboratory of Pathology, Research Center of Hospital de Clínicas de Porto Alegre, Rio Grande do Sul, Brazil; ${ }^{4}$ Department of Pathology, Universidade Federal do Rio Grande do Sul, Rio Grande do Sul, Brazil; ${ }^{5}$ Gene Therapy Center, Research Center, Hospital de Clínicas de Porto Alegre, Rio Grande do Sul, Brazil; ${ }^{6}$ Department of Gastroenterology and Hepatology, Universidade Federal do Rio Grande do Sul, Rio Grande do Sul, Brazil. Correspondence: Jorge Luiz dos Santos (kapars@terra.com.br) 
life; there was no statistical difference related to age between the two groups $(P=0.381)$. The age group of most of the BA patients ( $n=13,56.5 \%$ ) was $60-89 \mathrm{~d}$ of life; in eight (35.0\%) patients, age at the procedure was $<60 \mathrm{~d}$, and in only two (8.5\%) patients, surgery was undertaken after $90 \mathrm{~d}$ of life.

\section{Gene Expression}

The gene expression of ANGPT1, evaluated by exponential $\Delta \Delta \mathrm{CT}$, in the BA group corresponded to 92.9 (range: $2.1-$ 23493.9), whereas in the IHC group, it was 14.6 (range: 1.0$32.5)$, showing a significant difference between these groups $(P$ $=0.024$; Figure 1 ).

ANGPT2 gene expression was 136.0 (range: $0.1-14076.5$ ) in the BA group and 14.6 (range: 1.0-32.7) in the IHC group, and these values were significantly different $(P=0.029$; Figure 2$)$. There was no statistical difference in the TIE2 gene expression between BA (3444.3 (range: 9.5-90147.5)) and IHC (10369.1 (range: 1.0-25521.7)) groups $(P=0.501)$.

\section{Morphometric Evaluations}

Area of collagen deposition (ACOL), which analyzes the extent of fibrosis in patients with $\mathrm{BA}$, was $24.1 \%$ (range: $2.6-38.7 \%$ ) in $\mathrm{BA}$, whereas in IHC, it was $13.1 \%$ (range: 5.3-29.6\%); no significant difference was observed between the two groups $(P=0.275)$. The percentage of CK7 positivity (PCK7), used to analyze the extent of ductular reaction, was 7.5\% (range: 1.7$15.1 \%$ ) in the BA group and 3.1\% (range: $2.3-5.4 \%$ ) in the IHC group; no significant difference was observed between the two groups $(P=0.065)$, although infants with BA seemed to have higher values (Table 1 ).

\section{Correlation Between Gene Expression and Variables Associated With Disease Severity}

When evaluating the interdependence between the expression levels of the ANGPT/TIE2 system, the ratio of medial

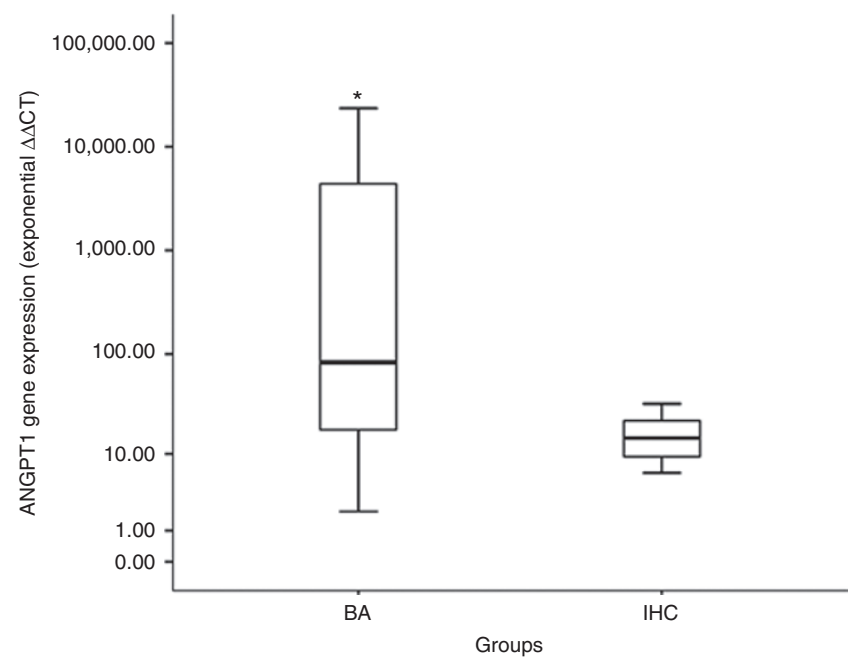

Figure 1. Expression of Angiopoietin 1 (ANGPT1) in patients with biliary atresia (BA) and intrahepatic cholestasis (IHC). Data are presented as median and interquartile range. Statistical analysis was performed by Mann-Whitney test. ${ }^{*} P=0.024$. thickness to luminal diameter (RMED) - which describes MT-and the variables associated with disease severity (age at portoenterostomy, ACOL, and PCK7) specifically in the BA group, the only parameter that showed correlation with the expression of ANGPT1 and ANGPT2 was RMED; in both the cases, a positive moderate correlation (ANGPT1: $r_{\mathrm{s}}=0.59, P$ $=0.013$; ANGPT2: $\left.r_{\mathrm{s}}=0.52, P=0.032\right)$ was identified. There were no correlations between the variables associated with disease severity (age at portoenterostomy, ACOL, or PCK7) and the expression of the ANGPT/TIE2 system. An inverse correlation between the expression of TIE2 and that of ANGPT1 and ANGPT2 was observed (ANGPT1: $r_{\mathrm{s}}=-0.73, P<0.001$; ANGPT2: $\left.r_{\mathrm{s}}=-0.54, P=0.007\right)$. Regarding variables associated with disease severity in BA, PCK7 was positively correlated both with age at portoenterostomy $\left(r_{s}=0.70, P=0.002\right)$ and ACOL $\left(r_{\mathrm{s}}=0.55, P=0.022\right.$; Table 2$)$.

Furthermore, we divided the BA group into subsets of high and low ANGPT1 expression taking into account the limit level of three times the value of maximal expression of this molecule within the IHC group and compared the MT evaluated by RMED between them. Using this approach, we observed that BA patients with high ANGPT1 expression had RMED values of 1.2 (range: 0.5-2.8), whereas those with low ANGPT1 expression showed RMED values of 0.9 (range: $0.4-1.5$ ), with a borderline statistical significance $(P=0.068$; Figure 3$)$.

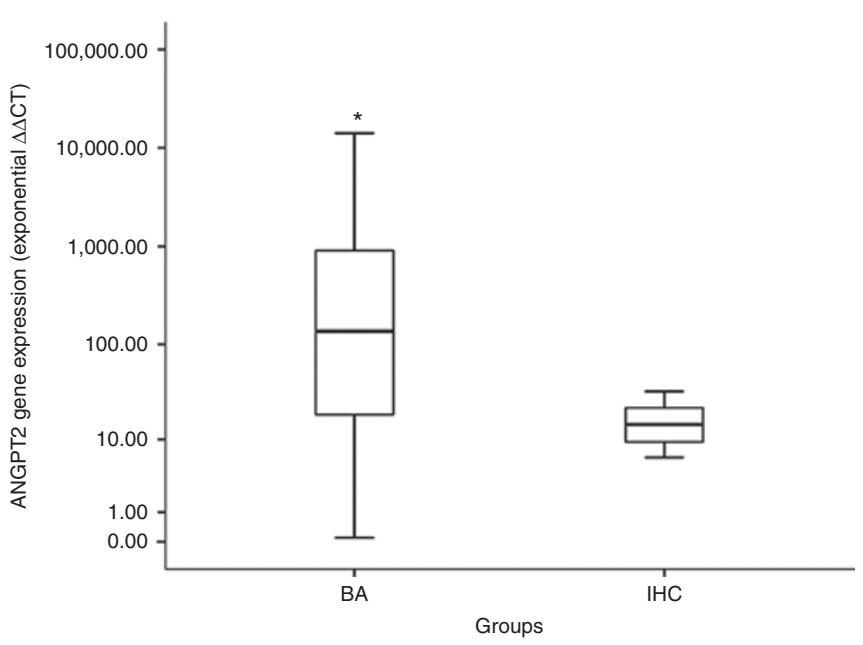

Figure 2. Expression of Angiopoietin 2 (ANGPT2) in patients with biliary atresia (BA) and intrahepatic cholestasis (IHC). Data are presented as median and interquartile range. Statistical analysis was performed by Mann-Whitney test. ${ }^{*} P=0.029$.

Table 1. Comparison between the biliary atresia and intrahepatic cholestasis groups in terms of the ACOL and PCK7

\begin{tabular}{lccc}
\hline & \multicolumn{2}{c}{ Group median (minimum to maximum) } & \\
\cline { 2 - 3 } Variables & Biliary atresia & Intrahepatic cholestasis & $P$ \\
\hline ACOL (\%) & $24.11(2.6-38.7)$ & $13.05(5.32-29.62)$ & 0.275 \\
PCK7 (\%) & $7.45(1.74-15.05)$ & $3.05(2.3-5.35)$ & 0.065 \\
\hline
\end{tabular}

Statistics according to Mann-Whitney test.

ACOL, area of collagen deposition; PCK7, percentage of CK7 positivity. 
Table 2. Correlations among ANGPT/TIE2 system gene expression, RMED, and variables associated with disease severity (AGEP, ACOL, and PCK7)

\begin{tabular}{lcccccc}
\hline Variables & ANGPT1 & ANGPT2 & TIE2 & RMED & PCK7 & ACOL \\
\hline ANGPT2 & 0.69 & - & & & & \\
& $(<0.001)$ & & & & & \\
TIE2 & -0.73 & -0.54 & - & & & \\
& $(<0.001)$ & $(0.007)$ & & & & \\
RMED & 0.59 & 0.52 & -0.46 & - & & \\
& $(0.013)$ & $(0.032)$ & $(0.063)$ & & & \\
PCK7 & -0.15 & 0.09 & 0.23 & 0.33 & - & \\
& $(0.955)$ & $(0.743)$ & $(0.384)$ & $(0.202)$ & & \\
ACOL & 0.06 & -0.07 & 0.14 & 0.39 & 0.55 & - \\
& $(0.808)$ & $(0.779)$ & $(0.599)$ & $(0.125)$ & $(0.022)$ & \\
AGEP & 0.18 & -0.02 & 0.05 & 0.03 & 0.70 & 0.33 \\
& $(0.428)$ & $(0.934)$ & $(0.805)$ & $(0.907)$ & $(0.002)$ & $(0.195)$ \\
\hline
\end{tabular}

Statistics based on Spearman correlation. Data are presented as the Spearman correlation coefficient and its statistical significance (within parentheses). Empty cells represent correlations displayed at the complementary sites of the table. (-) means correlation of one parameter with itself.

ACOL, area of collagen deposition; AGEP, age at portoenterostomy; ANGPT, angiopoietin; PCK7, percentage of CK7 positivity; RMED, ratio of medial thickness to luminal diameter; TIE2, tyrosine kinase with immunoglobulin-like and epidermal growth factor-like domains 2.

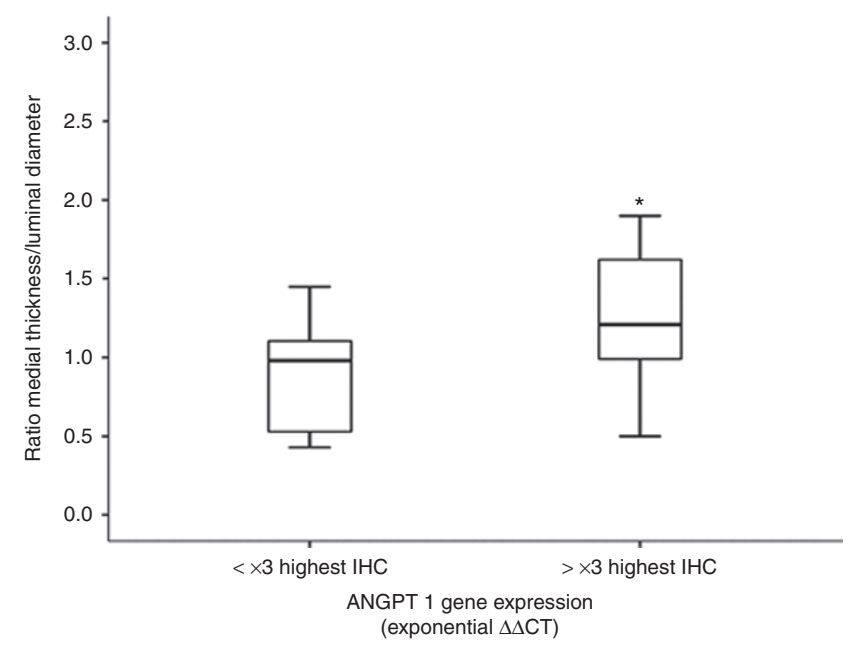

Figure 3. Comparison of the values of the ratio "medial thickness/luminal diameter" between the biliary atresia (BA) subsets with high and low expression of Angiopoietin 1 (ANGPT1), evaluated as exponential $\Delta \Delta C T$, considering the limit level of three times the value of maximal expression of this molecule within the intrahepatic cholestasis (IHC) group as the cutoff point. Data are presented as median and interquartile range. Statistical analysis was performed by Mann-Whitney test. ${ }^{*} P=0.068$.

\section{Effect of the Studied Variables on the Postoperative Outcome After Portoenterostomy}

In this study, 10 of 23 (43.5\%) patients died or underwent liver transplantation within $2 \mathrm{y}$ after portoenterostomy, with an age of 309 (range: 189-512) d. Survival with native liver at 2 y was $46.5 \%$. No studied variable, including ANGPT/TIE2 system expression, age at portoenterostomy, RMED, PCK7, and ACOL, influenced the occurrence of death and/or liver transplantation within the second postoperative year after portoenterostomy (Table 3).
Table 3. Association of studied variables and loss of native liver (transplantation or death) at $2 \mathrm{y}$ after portoenterostomy

\begin{tabular}{llcc}
\hline & \multicolumn{3}{l}{ Occurrence of death and/or liver transplantation } \\
\cline { 2 - 4 } Variables & $\mathrm{HR}$ & $95 \% \mathrm{Cl}$ & $\mathrm{P}$ \\
\hline ANGPT1 & 0.88 & $(0.59-1.33)$ & 0.553 \\
ANGPT2 & 1.2 & $(0.72-2.01)$ & 0.491 \\
TIE2 & 0.84 & $(0.44-1.57)$ & 0.576 \\
RMED & 2.5 & $(0.08-84.44)$ & 0.599 \\
ACOL & 1.02 & $(0.09-12.30)$ & 0.988 \\
PCK7 & 1.32 & $(0.09-19.84)$ & 0.843 \\
AGEP & 1.01 & $(0.98-1.05)$ & 0.428 \\
\hline
\end{tabular}

Statistics based on Cox regression, with hazard ratios (HRs) and $95 \%$ confidence intervals (Cls). Data were log-transformed before analysis.

ACOL, area of collagen deposition; AGEP, age at portoenterostomy; ANGPT, angiopoietin; PCK7, percentage of CK7 positivity; RMED, ratio of medial thickness to luminal diameter; TIE2, tyrosine kinase with immunoglobulin-like and epidermal growth factor-like domains 2.

Nine of 23 (39\%) patients were jaundice free at 6 mo after portoenterostomy. Clearance of jaundice in the first 6 mo following the surgical procedure was not affected by any studied variable (Table 4 ).

\section{Histological Immunolocalization of ANGPT1}

The immunolocalization of ANGPT1 expression occurred in arterial, venous, and sinusoidal endothelium in $100 \%$ of both the study groups. It was also detected in the medial layer and biliary structures from, respectively, 14 and $50 \%$ of the BA patients, whereas in only one patient with IHC, the bile ductules were positively stained (Figure 4).

\section{DISCUSSION}

Angiogenesis takes part not only during normal embryogenesis but also during the pathogenesis of diverse disorders, including liver diseases. Oxygen plays a central role in regulating angiogenesis, and vascular endothelial growth factor (VEGF) A, a major angiogenic factor, whenever induced by hypoxia, gives rise to new vessels aiming at restituting adequate tissue oxygenation (11). In the liver, fibrogenesis and angiogenesis are closely related processes. Cirrhosis deranges the normal hepatic vascularization and hepatocytes, limited within fibrotic nodules, suffer from hypoxia and sustain VEGF A production. Additionally, VEGF A induces ductular reaction and fibrogenesis (12). Mechanical architectural disturbances imposed by liver cell hyperplasia lead to blockade of hepatic vessels, perpetuating the cirrhogenic process.

Cholangiopathies show specific features with reference to blood vessels because bile ducts are nourished exclusively through the peribiliary vascular plexus formed by hepatic artery branches (13). Peribiliary vascular plexus is abnormal in disorders such as secondary sclerosing cholangitis (14) and some diseases are attributable to an ischemic cholangiopathy (15), whereas in many entities, the exact pathogenic role of an arterial disturbance remains unanswered $(16,17)$. BA encompasses a cholangiopathy of elusive nature affecting extra- and 
Table 4. Association of studied variables and clearance of jaundice (total bilirubin $<2 \mathrm{mg} / \mathrm{dl}$ ) at $6 \mathrm{mo}$ after portoenterostomy

\begin{tabular}{lccc}
\hline & \multicolumn{3}{c}{ Clearance of jaundice } \\
\cline { 2 - 4 } Variables & Yes & No & $P$ \\
\hline ANGPT1 & $97.1(2.7-4,770.8)$ & $48.5(2.1-13,216)$ & 0.673 \\
ANGPT2 & $162(2.7-6,905.4)$ & $48.5(0.1-1,644)$ & 0.387 \\
TIE2 & $2,896.3(268.7-80,126.9)$ & $3,444(9.5-90,147.5)$ & 0.730 \\
AGEP & $66(32-88)$ & $60(48-109)$ & 0.605 \\
RMED & $1.2(0.4-1.6)$ & $1.1(0.5-1.9)$ & 0.620 \\
PCK7 & $8.1(5.1-13.1)$ & $6.1(1.7-15.1)$ & 0.456 \\
ACOL & $19.8(14.3-37.4)$ & $25.3(2.6-38.7)$ & 0.620 \\
\hline
\end{tabular}

Statistics according to Mann-Whitney test. Data are presented as median (minimummaximum)

ACOL, area of collagen deposition; AGEP, age at portoenterostomy; ANGPT, angiopoietin; PCK7, percentage of CK7 positivity; RMED, ratio of medial thickness to luminal diameter; TIE2, tyrosine kinase with immunoglobulin-like and epidermal growth factor-like domains 2.
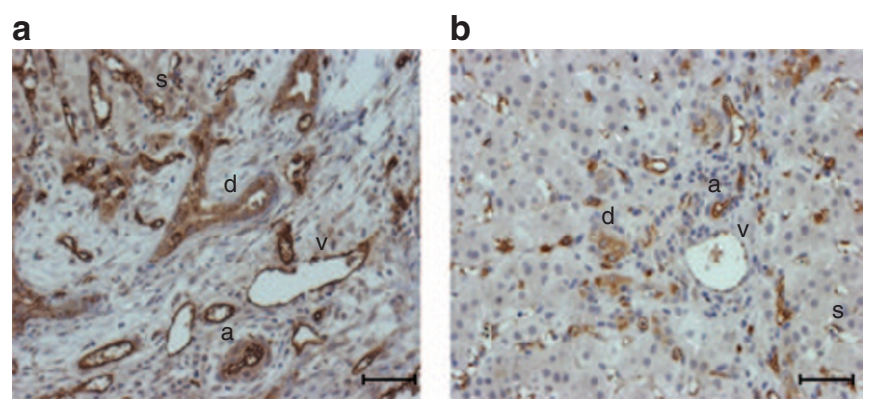

Figure 4. Immunolocalization of Angiopoietin 1 in patients under study (original magnification $\times 200$ ). Observe (a) positivity in venous $(v)$, arterial (a), and sinusoidal (s) endothelia, neoductules (d), and in the arterial medial layer (a) for the biliary atresia (BA) group; and (b) positivity in endothelia ( $\mathrm{v}, \mathrm{a}$, and $\mathrm{s}$ ) and in a neoductule (d), with absence of positivity in medial layer (a) for the intrahepatic cholestasis (IHC) group. Scale bar $=50 \mu \mathrm{m}$.

intrahepatic bile ducts, which generally results in cirrhosis irrespective of timely provision of portoenterostomy (1).

Our group evaluated the role of arteriopathy in BA etiology because affected patients show MT in hepatic arterial branches, progressive from portoenterostomy to liver transplantation. In porta hepatis, arteries show thickened and distorted walls $(8,9)$. In $\mathrm{BA}$, there are early arteriographic evidences of dystrophic peripheral hepatic artery branches, with irregularities in contour and images suggestive of occlusion. Angiographic vascular "tuft-like" blush surrounding occluded peripheral arterial segments are common (3). Ultrasound and computed tomography examinations demonstrate an enlarged hepatic arterial lumen at porta hepatis that is indicative of BA early on at the time of diagnosis and is suggestive of a blockade to the incoming blood flow located in intrahepatic vessels (4-7). Additionally, immunohistochemical expression of VEGF A in the liver of affected infants is localized in bile ducts and arterial walls, both in portal tracts and at porta hepatis, suggesting presence of hypoxia in such structures. VEGF A positivity at portoenterostomy is correlated with MT (9). Mechanisms and pathophysiological implications of an arterial vascular abnormality in BA must be thoroughly investigated, with the aim of defining the role of an ischemic cholangiopathy in its etiology.

In both normal and pathological conditions, following the initial vasculogenesis induced by VEGF, neovessels undergo additional steps for maturing and stabilizing the vascular walls, through the action of molecules such as the ANGPT/TIE2 system. ANGPT1 induces phosphorylation of the TIE2 receptor, thus promoting its activation, whereas ANGPT2 competitively inhibits TIE2 $(18,19)$. ANGPT1 promotes interaction between endothelial and underlying supporting cells, leading to recruitment of pericytes and smooth muscle cells to form support for neovessels. It is also involved in decreasing inflammation and vascular permeability (20), acting synergistically with VEGF not only in angiogenesis but also in immunity to integrate inflammatory and angiogenic responses. Moreover, cytokines induced by ANGPT1 stimulate the expression of VEGF, thus potentiating tissue neovascularization (21). In animal models, overexpression of both ANGPT1 and VEGF leads to proliferation of enlarged vessels in skin (22). Isolated ANGPT1 overexpression in rats leads to pulmonary hypertension owing to MT in small arteries, and lung angiograms of the affected animals show blunting of peripheral arterioles (23). Because these findings fit what has been observed in BA by liver histology (8) and arteriography (3), we performed this study aiming to assess the expression of ANGPT1 in liver from affected patients.

We observed that in BA patients, there is overexpression of ANGPT1 in comparison with IHC and that the expression of this molecule is correlated with MT. Additionally, dividing the BA group into the two subsets with high and low expression of ANGPT1, we found that MT seemed to be different between these subgroups.

In the immunohistochemical evaluation, ANGPT1 was expressed in vascular and sinusoidal endothelia in patients with IHC, whereas in $50 \%$ of the infants with BA, it was present, apart from this usual localization, in the biliary epithelium. Fabris et al. (24) observed ANGPT1 expression in cholangiocytes of patients with autosomal dominant polycystic kidney disease, which includes a biliary malformation disorder, and in the normal embryonic ductal plate. In autosomal dominant polycystic kidney disease, ANGPT1 in concert with VEGF induced proliferation of cholangiocytes and vasculature, thus promoting the growth of cysts and their vascular supply (24). In this study, in addition, we observed ANGPT1 immunolocalization in the medial layers in $14 \%$ of the liver samples from patients with BA, and this specific pattern of ANGPT1 expression may be displaying the vascular abnormality in BA associated with MT. The insult that promotes MT in BA is elusive and may involve local immune-mediated processes, local gene expression alterations, or even the prolonged increased shear stress attributable to the intrahepatic portal hypertension present in the disease. The endothelial dysfunction caused by any of these putative mechanisms may result in MT by inducing secretion of molecules such as ANGPTs. Irrespective to the cause of MT, this abnormality could result in ischemic cholangiopathy and the associated bile duct paucity and/or the secondary sclerosing cholangitis commonly observed in BA. 


\section{Articles $\mid$ de Souza e tal.}

An unexpected finding in this study was the occurrence of ANGPT2 overexpression, also correlated with MT, in BA. Formerly, ANGPT2 was thought to have only a competitive role to ANGPT1 with reference to TIE2. Presently, it is known that ANGPT2 acts on neovessel maturation depending on tissue milieu (25), particularly the expression of VEGF A. In the presence of VEGF A, ANGPT2 potentiates the maturational activity of ANGPT1 on neovessels, whereas in its absence, ANGPT2 competes with ANGPT1, thus promoting vascular regression $(26,27)$. Additionally, the interaction between ANGPT2 and VEGF plays a role in both liver fibrosis (28) and hepatocellular carcinoma (29). Our findings on the additive effect of ANGPT1 and ANGPT2 on MT in patients with BA may be explained by the fact that VEGF A is strongly expressed in portal structures, including the medial layer of hepatic artery branches in these patients (9).

A second unexpected finding in this study was the negative correlation between the expression of both ANGPTs and that of the TIE2 receptor. This may result from the competitive balance between VEGF and ANGPT1 that occurs in proangiogenic states to avoid a complete vascular stabilization, thus maintaining neovessel sprouting. This is achieved through the cleavage of TIE2 by VEGF, creating a soluble TIE2, which, on binding to both ANGPTs, inhibits downstream signaling and regulates vessel maturation (21). A similar process may have affected the tissue levels of the TIE2 receptor in the liver of our BA patients, which probably shows a pronounced proangiogenic state owing to the fibrogenic process. Another mechanism for attenuating TIE2 activation is the induction of its turnover in a dose-dependent manner by ANGPT1 and, less intensely, by ANGPT2, leading to the ubiquitination and degradation of the receptor (30). The amplitude and length of the signaling over tyrosine kinase receptors such as TIE2 seem to have a critical role in such a mechanism of attenuation (31). The high levels of ANGPT expression, associated with the chronic nature of $\mathrm{BA}$, may explain the negative correlation between ANGPT1 and ANGPT2 and their receptor TIE2 in the affected patients. However, aiming to fully elucidate how ANGPTs can induce MT in the presence of normal values of TIE2 and even showing a negative correlation with the expression of this receptor, it will be necessary to evaluate the role of a recently described receptor of ANGPT1 named TIE1, whose overexpression is associated with atherosclerosis, increased shear stress, and inflammation (32).

In addition, a third unexpected finding was the absence of correlation between the expression of both ANGPTs and the variables associated with disease severity in BA (age at portoenterostomy, ACOL, and PCK7) because there are reports linking these angiogenic molecules to hepatic fibrogenesis and, hence, to disease aggravation (33). Specifically in the context of ACOL, this lack of association may be ascribed to inherent difficulties in the analysis of liver fibrosis, such as sampling errors and phenotypic idiosyncratic features in relation to fibrogenesis (2). The absence of correlation between the expression of ANGPTs and the extent of fibrosis goes against the supposition that the overexpression of these molecules in BA, and the associated
MT, can be fully explained by increased levels of shear stress. However, the small number of BA patients under study may also account for this negative finding and for the absence of correlation between expression of ANGPTs and the other variables associated with disease severity, age at portoenterostomy, and PCK7. Our findings suggest that in BA, the overexpression of both ANGPT1 and ANGPT2 is not related to the clinical-histological severity of liver disease at the time of portoenterostomy.

What does the overexpression of ANGPTs associated with MT that we observe in BA mean? Desmet (34) described the occurrence of venous hypoplasia associated with the arterial vessel hyperplasia in liver samples from patients with BA. A putative explanation to his finding and to our own observations is that we are detecting a vascular alteration secondary to the intrahepatic portal hypertension in BA. In situations of intense liver fibrosis, the hepatic blood flow becomes increasingly arterialized. In BA, a disease characterized by early intense fibrosis and portal hypertension, this may be particularly pronounced, leading to an imbalance between angiogenic factors involved with the arterial-venous specification and determining the histological features observable in the liver such as decreased number of portal venous branches associated with an increased amount of arterial vessels. Portal vein hypoplasia at the liver hilum could be a secondary event to decreased venous blood flow, and heightened shear stress could explain MT. VEGF and ANGPTs would be the signaling molecules involved in such a pathophysiological process. However, the absence of correlation between expression of ANGPTs and the variables associated with disease severity, including extent of fibrosis, does not allow us to embrace this view. Another explanation is that in BA, there is a primary altered expression of genes that control the arterial/venous specification and vascular maturation such as Sonic Hedgehog or Notch by affecting angiogenic molecules such as ANGPTs $(35,36)$. In this case, our findings could be reflecting the downstream molecular effect of a local gene dysregulation.

In conclusion, this study shows that in BA, there is overexpression of both ANGPT1 and ANGPT2, which is correlated with MT and occurs irrespective of both age at portoenterostomy and histological variables associated with disease severity (extents of fibrosis and ductular reaction) at the time of the procedure.

\section{METHODS}

This study included infants with BA $(n=23)$ and IHC $(n=7)$, evaluated at the time of the exploratory laparotomy that preceded portoenterostomy in $\mathrm{BA}$, or excluded this diagnosis in the remaining cases, presenting a clinical picture that, otherwise, could not be distinguished. The causes of IHC encompassed idiopathic neonatal hepatitis $(n=4)$ and $\alpha-1$ antitrypsin deficiency $(n=3)$. During the procedure, two tissue samples were collected from segment IV of the liver. The first was immediately placed in a tube containing $1 \mathrm{ml}$ of RNA holder (BioAgency, São Paulo, Brazil) and sequentially stored in a freezer at $-80^{\circ} \mathrm{C}$ for RNA expression assays. The second tissue sample was kept in formalin and subsequently embedded in paraffin for future histological analyses.

\section{Procedures of Quantitative PCR}

RNA Extraction and cDNA Generation. RNA was extracted from $50 \mathrm{mg}$ of tissue using Brazol (Labtrade, São Paulo, Brazil). Briefly, samples were macerated in $1 \mathrm{ml}$ of Brazol. Then, cDNA was generated from 
$3 \mu \mathrm{g}$ of RNA using the cDNA Superscript II e III RT Kit (Invitrogen, Carlsbad, CA), following the manufacturer's instructions.

Quantitative PCR. Quantitative PCR was performed using the SYBRGreenER SuperMix Universal kit (Invitrogen). Primers, their annealing temperatures, and amplicon sizes for ANGPT1, ANGPT2, TEK (TIE2 receptor), and the control gene RNA18S are depicted in Table 5. PCR reaction was conducted using StepOne (Life Technologies, Carlsbad, CA), and relative quantification was done using the $2-\Delta \Delta \mathrm{CT}$ method.

\section{Morphometric Assessments of Histological Variables}

Sample preparation and image capture. From each paraffin-embedded liver tissue sample, $5-\mu \mathrm{m}$ sections were obtained for staining with hematoxylin and eosin, picrosirius red, and by immunohistochemistry, for cytokeratin 7 (CK7; Dako, Glostrup, Denmark; dilution, 1:100) and ANGPT1 (R\&D Systems, Minneapolis, MN; dilution 1:100). Immunolabeling was amplified using the avidin-biotin-peroxidase complex, as described previously (37), the secondary antibody being a multispecies reagent (EasyPath; Erviegas, São Paulo, Brazil). Slides stained with hematoxylin and eosin were used for the morphometric assessment of the RMED to describe MT, whereas those stained with picrosirius red were used for assessing the ACOL, with the aim of analyzing the extent of liver fibrosis. Staining with CK7 was used for quantification of PCK7 to evaluate the extent of ductular reaction. Images were captured under an Olympus BX51 photomicroscope equipped with a digital camera (Olympus BX60). For morphometric measurement, 10 images from each slide were captured from randomly selected high-power fields $(\times 200$ magnification) containing hepatic artery branches (in slides stained with hematoxylin and eosin), fibrotic areas (in picrosirius red-stained slides), and CK7-positive structures. Morphometric measurements were performed using the Adobe Photoshop CS3 Extended 10.0 (Adobe Systems, San Jose, CA). A total of 10 images from each patient sample were analyzed. The histological assessor in the morphometric studies was blinded to clinical data.

\section{Morphometric Calculations}

RMED. RMED was evaluated as previously described (8). Both MT and luminal diameter were analyzed using the manual measurement tool of the digital analysis software. For each vessel, MT was calculated as the average of six length measurements of arterial muscular wall, each one obtained by a line drawn to the central axis and connecting one point placed at the media-adventitia interface and another situated at the media-intima interface. Luminal diameter was calculated as the average of length measures of two lines, each one drawn between two opposite points both of them placed at the media-intima interface and assessing the major and the minor diameters.

PCK7. PCK7 assessment was performed as described elsewhere (2). PCK7 was measured in pixels through the color range tool of the

Table 5. Primers used in this study, their temperatures of annealing, and molecular sizes

\begin{tabular}{llcc}
\hline Molecules & \multicolumn{1}{c}{ Primers } & AT & Size (bp) \\
\hline ANGPT1 & F: GGTCACACTGGGACAGCAG & $58{ }^{\circ} \mathrm{C}$ & 134 \\
& R: CATCAAACCACCATCCTCCTG & $58{ }^{\circ} \mathrm{C}$ & \\
ANGPT2 & F: GCAGCATCAGCCAACCAGG & $59.5^{\circ} \mathrm{C}$ & 106 \\
& R: GCATCAAACCACCAGCCTCC & $59.0^{\circ} \mathrm{C}$ & \\
TIE2 & F: CGGAGATGTGAAGCCCAGAAG & $57.8^{\circ} \mathrm{C}$ & 123 \\
& R: CCTTCCATAAACCCAGGAGG & $57.3^{\circ} \mathrm{C}$ & \\
RNA18S & F: GACGGACCAGAGCGAAAGC & $58.6^{\circ} \mathrm{C}$ & 113 \\
& R: GTCGGCATCGTTTATGGTCGG & $58.4^{\circ} \mathrm{C}$ & \\
\hline
\end{tabular}

ANGPT, angiopoietin; AT, annealing temperature; bp, base pairs; $F$, forward primer; R, reverse primer; RNA18S, 18 S ribosomal RNA; TIE2, tyrosine kinase with immunoglobulin-like and epidermal growth factor-like domains 2. image analysis software, selecting a foreground color and a background color and then applying the Grow tool to amplify the spectrum of the color of interest so that the whole CK7-positive area was represented. Total amount of pixels per image remained constant in all fields for all the samples analyzed. PCK7 in each image was calculated using the ratio "CK7-positive area per total amount of pixels per image."

$A C O L$. ACOL quantification was undertaken as previously described (38). The ACOL was calculated using the ratio "picrosirius red-positive area per total amount of pixels per image." The total amount of pixels per image remained constant in all fields for all the samples analyzed. For each image, we used the color range tool to choose a foreground color including a picrosirius red-positive area and a background color encompassing the counterstained surface and then applied the Grow tool so that the entire picrosirius red-positive area was selected.

\section{Immunolocalization of ANGPT1}

Histological immunolocalization of ANGPT1 expression was performed by an expert in liver pathology (L.M.), blinded to clinical data, making use of a previously described semiquantitative method (39).

\section{Evaluation of Postoperative Outcome in Patients With BA}

Outcome after portoenterostomy was evaluated by clearance of jaundice in the first six postoperative months and by analyzing the loss of native liver in terms of transplantation or death at $2 \mathrm{y}$ following the procedure. Clinical data were prospectively collected by the authors involved in patient clinical care.

\section{Statistical Analysis}

Quantitative variables were expressed as mean \pm SD or median (range), and categorical data were described as frequencies and percentages. Student's $t$-test or Mann-Whitney tests were used for comparing groups according to the data symmetry. Interdependence among variables was evaluated by the Spearman correlation test.

Association of the studied variables with loss of native liver was evaluated by Cox regression, with hazard ratios and $95 \%$ confidence intervals. Additionally, we used Fisher exact procedure to evaluate the effect of studied variables on loss of native liver. Data were logtransformed before analysis.

A $P$ value $<0.05$ was considered significant. The softwares Microsoft Excel 2007 (Microsoft, Redmond, WA) and SPSS 18.0 (SPSS, Chicago, IL) were used for data processing and analysis.

\section{Ethics Statement}

Informed consent for the use of histological specimens and clinical data were obtained from patients' parents or guardians. The Research and Postgraduation Group Ethics Committee of the Hospital de Clínicas de Porto Alegre approved this study.

\section{ACKNOWLEDGMENTS}

This study was part of the Master's degree thesis of A.F.d.S., presented to the Postgraduate Course in Child and Adolescent Health, Universidade Federal do Rio Grande do Sul, Brazil. We acknowledge Jorge Abdon Bezerra, from the Cincinnati Children's Hospital Medical Center, Cincinnati, Ohio, for his valuable support in the inaugural steps of this study by screening candidate angiogenic molecules through a transcriptome analysis in patients with neonatal cholestasis.

\section{STATEMENT OF FINANCIAL SUPPORT}

We acknowledge the financial support of Fundo de Incentivo à Pesquisa e Eventos (FIPE) from Hospital de Clínicas de Porto Alegre, Rio Grande do Sul, Brazil.

Disclosure: Authors have no conflict of interests to disclose.

\section{REFERENCES}

1. Santos JL, Carvalho E, Bezerra JA. Advances in biliary atresia: from patient care to research. Braz J Med Biol Res 2010;43:522-7.

2. Santos JL, Kieling CO, Meurer L, et al. The extent of biliary proliferation in liver biopsies from patients with biliary atresia at portoenterostomy is associated with the postoperative prognosis. J Pediatr Surg 2009;44:695-701. 
3. Uflacker R, Pariente DM. Angiographic findings in biliary atresia. Cardiovasc Intervent Radiol 2004;27:486-90.

4. Humphrey TM, Stringer MD. Biliary atresia: US diagnosis. Radiology 2007;244:845-51.

5. Kim WS, Cheon JE, Youn BJ, et al. Hepatic arterial diameter measured with US: adjunct for US diagnosis of biliary atresia. Radiology 2007;245:549-55.

6. Lee MS, Kim MJ, Lee MJ, et al. Biliary atresia: color doppler US findings in neonates and infants. Radiology 2009;252:282-9.

7. Caruso S, Miraglia R, Milazzo M, et al. Multidetector computed tomography hepatic findings in children with end-stage biliary atresia. Eur Radiol 2010;20:1468-75.

8. dos Santos JL, da Silveira TR, da Silva VD, Cerski CT, Wagner MB. Medial thickening of hepatic artery branches in biliary atresia. A morphometric study. J Pediatr Surg 2005;40:637-42.

9. Edom PT, Meurer L, da Silveira TR, Matte U, dos Santos JL. Immunolocalization of VEGF A and its receptors, VEGFR1 and VEGFR2, in the liver from patients with biliary atresia. Appl Immunohistochem Mol Morphol 2011;19:360-8.

10. Jin SW, Patterson C. The opening act: vasculogenesis and the origins of circulation. Arterioscler Thromb Vasc Biol 2009;29:623-9.

11. Rosmorduc O, Wendum D, Corpechot C, et al. Hepatocellular hypoxiainduced vascular endothelial growth factor expression and angiogenesis in experimental biliary cirrhosis. Am J Pathol 1999;155:1065-73.

12. Gaudio E, Barbaro B, Alvaro D, et al. Vascular endothelial growth factor stimulates rat cholangiocyte proliferation via an autocrine mechanism. Gastroenterology 2006;130:1270-82.

13. Kobayashi S, Nakanuma Y, Matsui O. Intrahepatic peribiliary vascular plexus in various hepatobiliary diseases: a histological survey. Hum Pathol 1994;25:940-6.

14. Gelbmann CM, Rümmele P, Wimmer M, et al. Ischemic-like cholangiopathy with secondary sclerosing cholangitis in critically ill patients. Am J Gastroenterol 2007;102:1221-9.

15. Deltenre P, Valla DC. Ischemic cholangiopathy. J Hepatol 2006;44:806-17.

16. Kono N, Nakanuma Y. Ultrastructural and immunohistochemical studies of the intrahepatic peribiliary capillary plexus in normal livers and extrahepatic biliary obstruction in human beings. Hepatology 1992;15:411-8.

17. Washington K, Clavien PA, Killenberg P. Peribiliary vascular plexus in primary sclerosing cholangitis and primary biliary cirrhosis. Hum Pathol 1997;28:791-5.

18. Davis S, Aldrich TH, Jones PF, et al. Isolation of angiopoietin-1, a ligand for the TIE2 receptor, by secretion-trap expression cloning. Cell 1996;87:1161-9.

19. Suri C, Jones PF, Patan S, et al. Requisite role of angiopoietin-1, a ligand for the TIE2 receptor, during embryonic angiogenesis. Cell 1996;87:1171-80.

20. Nykänen AI, Pajusola K, Krebs R, et al. Common protective and diverse smooth muscle cell effects of AAV-mediated angiopoietin- 1 and -2 expression in rat cardiac allograft vasculopathy. Circ Res 2006;98:1373-80.

21. Novotny NM, Lahm T, Markel TA, et al. Angiopoietin-1 in the treatment of ischemia and sepsis. Shock 2009;31:335-41.
22. Thurston G, Suri C, Smith K, et al. Leakage-resistant blood vessels in mice transgenically overexpressing angiopoietin-1. Science 1999;286:2511-4.

23. Chu D, Sullivan CC, Du L, et al. A new animal model for pulmonary hypertension based on the overexpression of a single gene, angiopoietin-1. Ann Thorac Surg 2004;77:449-56; discussion 456-7.

24. Fabris L, Cadamuro M, Fiorotto R, et al. Effects of angiogenic factor overexpression by human and rodent cholangiocytes in polycystic liver diseases. Hepatology 2006;43:1001-12.

25. Teichert-Kuliszewska K, Maisonpierre PC, Jones N, et al. Biological action of angiopoietin-2 in a fibrin matrix model of angiogenesis is associated with activation of Tie2. Cardiovasc Res 2001;49:659-70.

26. Holash J, Wiegand SJ, Yancopoulos GD. New model of tumor angiogenesis: dynamic balance between vessel regression and growth mediated by angiopoietins and VEGF. Oncogene 1999;18:5356-62.

27. Holash J, Maisonpierre PC, Compton D, et al. Vessel cooption, regression, and growth in tumors mediated by angiopoietins and VEGF. Science 1999;284:1994-8.

28. Scholz A, Rehm V, Rieke S, et al. Angiopoietin-2 serum levels are elevated in patients with liver cirrhosis and hepatocellular carcinoma. Am J Gastroenterol 2007;102:2471-81.

29. Yoshiji H, Kuriyama S, Noguchi R, et al. Angiopoietin 2 displays a vascular endothelial growth factor dependent synergistic effect in hepatocellular carcinoma development in mice. Gut 2005;54:1768-75.

30. Wehrle C, Van Slyke P, Dumont DJ. Angiopoietin-1-induced ubiquitylation of Tie 2 by c-Cbl is required for internalization and degradation. Biochem J 2009;423:375-80.

31. Rubin C, Gur G, Yarden Y. Negative regulation of receptor tyrosine kinases: unexpected links to c-Cbl and receptor ubiquitylation. Cell Res 2005; $15: 66-71$.

32. Woo KV, Baldwin HS. Role of Tie1 in shear stress and atherosclerosis. Trends Cardiovasc Med 2011;21:118-23.

33. Taura K, De Minicis S, Seki E, et al. Hepatic stellate cells secrete angiopoietin 1 that induces angiogenesis in liver fibrosis. Gastroenterology 2008;135:1729-38.

34. Desmet VJ. Congenital diseases of intrahepatic bile ducts: variations on the theme "ductal plate malformation". Hepatology 1992;16:1069-83.

35. Kume T. Specification of arterial, venous, and lymphatic endothelial cells during embryonic development. Histol Histopathol 2010;25:637-46.

36. Kory J, Lavine K, Long F, et al. Hedgehog signaling to distinct cell types differentially regulates coronary artery and vein development. Development 2008; 135:3161-71.

37. Hsu SM, Raine L, Fanger H. Use of avidin-biotin-peroxidase complex (ABC) in immunoperoxidase techniques: a comparison between $\mathrm{ABC}$ and unlabeled antibody (PAP) procedures. J Histochem Cytochem 1981;29:577-80.

38. Masseroli M, Caballero T, O’Valle F, Del Moral RM, Pérez-Milena A, Del Moral RG. Automatic quantification of liver fibrosis: design and validation of a new image analysis method: comparison with semi-quantitative indexes of fibrosis. J Hepatol 2000;32:453-64.

39. Wu AW, Gu J, Li ZF, Ji JF, Xu GW. COX-2 expression and tumor angiogenesis in colorectal cancer. World J Gastroenterol 2004;10:2323-6. 